\title{
ON THE DESKINS INDEX COMPLEX OF A MAXIMAL SUBGROUP OF A FINITE GROUP
}

\author{
A. BALLESTER-BOLINCHES AND LUIS M. EZQUERRO
}

(Communicated by Warren J. Wong)

\begin{abstract}
We analyze the influence of the maximal subgroups in the structure of a finite group $G$ by means of the index complex introduced by Deskins in Proc. Sympos. Pure Math. vol. 1, Amer. Math. Soc. 1959, pp. 100-104.
\end{abstract}

\section{INTRODUCTION AND STATEMENTS OF RESULTS}

All groups considered are finite. In [4], Deskins defines the index complex associated to a maximal subgroup of a group as follows: let $M$ be a maximal subgroup of a group $G$; a subgroup $C$ of $G$ is said to be a completion for $M$ in $G$ if $C$ is not contained in $M$ while every proper subgroup of $C$ that is normal in $G$ is contained in $M$. The set $I(M)$ of all completions of $M$ is called the index complex of $M$ in $G$.

In [4], Deskins proved that the index complex of a maximal subgroup is nonempty. It is clear that if $C$ is a completion of $M$ in $G$ the product of all normal subgroups of $G$ that are proper subgroups of $C$ is itself a proper subgroup of $C$. Denote this subgroup by $k(C)$. A subgroup $C$ of a group $G$ is said to be a normal (respectively, subnormal) completion of a maximal subgroup $M$ of $G$ if $C$ is a normal (respectively, subnormal) subgroup of $G$ and $C \in I(M)$.

If $C$ and $D$ are normal completions of a maximal subgroup $M$ of a group $G$, then $C / k(C) \cong D / k(D)$; hence the order $|C / k(C)|$, where $C$ is a normal completion of $M$ in $G$, is an invariant depending only on $M$ and is called the normal index of $M$ in $G$. However, there is no invariant associated to subnormal completions.

Example. Let $P$ denote the extra-special group of order $3^{3}$ and $\exp P=3$ :

$$
P=\left\langle a, b, c: a^{3}=b^{3}=c^{3}=1,[a, c]=[b, c]=1,[a, b]=c\right\rangle \text {. }
$$

Let $G$ be the extra-special group of order $3^{7}$ and $\exp G=3$. $G$ is the central product of two copies $P_{1}, P_{2}$ isomorphic to $P$. Subindex the elements of $P_{1}$ and $P_{2}$ in the obvious way and keep this notation in $G$ with the identification

Received by the editors August 13, 1990.

1980 Mathematics Subject Classification (1985 Revision). Primary 20D10, 20D25.

Key words and phrases. Maximal subgroups, index complex.

This research was supported by DGICYT-proyecto no. PS87-0055-C02-02. 
$c_{1}=c_{2}=c$. Then $M=\left\langle a_{1}, b_{1}, c, a_{2}\right\rangle$ is a maximal subgroup of $G$, and obviously any completion of $M$ in $G$ is subnormal. It is not difficult to find $C_{1}, C_{2} \in I(M)$ with $\left|C_{1} / k\left(C_{1}\right)\right| \neq\left|C_{2} / k\left(C_{2}\right)\right|$. For instance, take $C_{1}=\left\langle b_{2}\right\rangle$ and $C_{2}=\left\langle b_{1}, b_{2}\right\rangle$.

It is clear that $I(M)$ can be partially ordered by set-theoretic inclusion; maximal elements of $I(M)$ are called maximal completions of $M$ in $G$.

In [5], Deskins proved that a group $G$ is soluble if and only if each maximal subgroup of $G$ has maximal completion $C$ with $C / k(C)$ abelian. In the same paper, he shows that the supersolubility cannot be characterized in the same way. Trying to characterize the supersolubility by means of the index complex, Deskins defines the following family associated to a maximal subgroup $M$ of a group $G: P(M)=\{C \in I(M): C$ is maximal in $I(M)$ and $G=C M\}$. He conjectures that a group $G$ is supersoluble if and only if for each maximal subgroup $M$ of $G, P(M)$ contains an element $C$ with $C / k(C)$ cyclic.

The answer to this conjecture is negative, as the symmetric group $S_{4}$ shows: $G=S_{4}$ has three conjugacy classes of maximal subgroups, namely, $\left\{A_{4}\right\}$, $\operatorname{Syl}_{2}(G)$, and $\left\{N_{G}\left(P_{3}\right): P_{3} \in \operatorname{Syl}_{3}(G)\right\}$. If $M=A_{4}$, then $G \in P(M), k(G)=$ $M$, and $G / k(G)$ is cyclic. If $M \in \operatorname{Syl}_{2}(G)$, then $A_{4} \in P(M), k\left(A_{4}\right)=V$, the 4-group of Klein, and $A_{4} / V \cong C_{3}$. Finally, if $M=N_{G}\left(P_{3}\right)$ with $P_{3} \in \operatorname{Syl}_{3}(G)$, then $C=\langle(1234)\rangle \in P(M)$, and obviously $C / k(C)=C$ is cyclic. However, $S_{4}$ is not supersoluble.

Let $M$ be a maximal subgroup of a group $G$. Let $S(M)=\{C \in I(M): C$ subnormal in $G$ and $G=M C\}$. Clearly, every normal completion of $M$ in $G$ is in $S(M)$. Hence $S(M)$ is nonempty. We prove

Theorem 1. A group $G$ is supersoluble if and only if for each maximal subgroup $M, S(M)$ contains an element $C$ with $C / k(G)$ cyclic.

On the other hand, one might wonder whether a group $G$ is soluble if each maximal subgroup $M$ has a maximal completion $C$ with $C / k(C)$ nilpotent. The answer is negative in general.

Consider the group $G=\operatorname{PGL}(2,17), G$ is a primitive group such that $\operatorname{Soc}(G)=\operatorname{PSL}(2,17)$. Furthermore $\operatorname{Soc}(G)$ is a maximal subgroup of $G$ of index 2; thus, $G$ is its maximal completion and $k(G)=\operatorname{Soc}(G)$, and then $G / k(G) \cong C_{2}$ is nilpotent. The other maximal subgroups of $G$ are core-free; a conjugacy class of these core-free maximal subgroups is $\operatorname{Syl}_{2}(G)$. Then any core-free maximal subgroup $M$ of $G$ has maximal completion $P \in \operatorname{Syl}_{2}(G)$ with $k(P)=1$, and then $P / k(P)$ is nilpotent.

Notice that the composition factors of $G$ are $C_{2}$ and $\operatorname{PSL}(2,17)$. In fact the only possibilities are of these types, as the next theorem shows.

Theorem 2. Assume that each maximal subgroup $M$ of a group $G$ has a maximal completion $C \in I(M)$ with $C / k(C)$ nilpotent. Then every composition factor of $G$ is in $\mathscr{X}$, where

$$
\mathscr{X}=\left\{C_{p}: p \in \mathbb{P}\right\} \cup\left\{\operatorname{PSL}(2, q): q=2^{n} \pm 1, q>5\right\} .
$$

Obviously the converse is not true; if $S$ is any simple group, the unique maximal completion of any maximal subgroup of $S$ is $S$ itself.

Now we consider the question: what do certain intrinsic properties of a maximal subgroup $H$ of a group $G$ imply about $G$ ? Deskins proved in [4] the 
following result: If a group $G$ contains a maximal subgroup $H$ which is supersoluble and if, for each $C \in I(H)$ with $C$ nonnormal or with $C \cap H$ nontrivial, $C / k(C)$ is supersoluble, then $G$ is soluble. We improve this result by proving

Theorem 3. Let $\pi$ be a set of primes. Suppose that $G$ is a group with a maximal subgroup $H$ which is $\pi$-soluble. Assume that for each completion $C \in I(H)$ such that $C \cap H \neq 1$, the factor $C / k(C)$ is $\pi$-soluble. Then $G$ is $\pi$-soluble.

If $M$ is a maximal subgroup of a group $G$ such that the index of $M$ in $G$ is composite, we say that $M$ is a $c$-maximal subgroup of $G$.

Definition. Let $G$ be a group and let $\mathscr{J}(G)=\{M: M$ is a $c$-maximal subgroup of $G$ and $M$ has no maximal completion $C$ with $C / k(C)$ abelian .

Define $S(G)=\bigcap\{M: M \in \mathscr{J}(G)\}$ if $\mathscr{J}(G)$ is nonempty; otherwise set $S(G)=G$.

It is clear from the definition that $S(G)$ is a characteristic subgroup of $G$ and $S(G)$ contains $\Phi(G)$.

The following result shows how $S(G)$ controls the solubility of a group $G$ :

Theorem 4. Let $G$ be a group. Then $S(G)$ is the soluble radical of $G$, i.e., the product of all soluble normal subgroups of $G$. In particular, $G$ is soluble if and only if each c-maximal subgroup of $G$ has a maximal completion $C$ with $C / k(C)$ abelian.

The motivation of our next result is as follows. Rose proved in [9] that if every abnormal subgroup of a group $G$ is $p$-nilpotent and if in addition either (i) the Sylow $p$-subgroups of $G$ are abelian or (ii) $p$ is an odd prime, then $G$ is $p$-soluble. We extend this result by proving

Theorem 5. Let $G$ be a group and $p$ a prime. Consider the family $\mathscr{A}(G)=$ $\{M: M$ is a maximal subgroup of $G$ and $M$ has no maximal completion $C$ with $C / K(C)$ abelian $\}$.

If each $M \in \mathscr{A}(G)$ is p-nilpotent and if in addition either (i) the Sylow $p$-subgroups of $G$ are abelian or (ii) $p$ is an odd prime, then $G$ is p-soluble.

\section{Preliminary Results}

The results given below are used frequently in induction arguments.

Proposition 1. Let $\mathscr{H}$ be a homomorph that is closed under taking normal subgroups. Assume $M$ is a maximal subgroup of a group $G$ and $N$ is a normal subgroup of $G$ such that $N \leq M$. If $C$ is a maximal (respectively, subnormal) completion of $M$ in $G$ with $C / k(C) \in \mathscr{H}$, there exists a maximal (respectively, subnormal) completion $C^{*}$ of $M$ in $G$ such that $N \leq C^{*}$ and $C^{*} / k\left(C^{*}\right) \in \mathscr{H}$.

Proof. Assume that $M$ has a maximal completion $C$ with $C / k(C) \in \mathscr{H}$. If $N \leq C$ take $C^{*}=C$ and we are done. So, we can assume $N \not C$. Since $C$ is a maximal completion of $M$, and $C<C N$, we have that $k(C N) \not \leq M$. This implies that there exists a chief factor $C^{*} / L$ of $G$ such that $k(C) N \leq$ $L<C^{*} \leq k(C N), L \leq M$ and $G=M C^{*}$. Therefore, $C^{*}$ is a maximal completion of $M, N \leq C^{*}$, and, since

$$
k(C N) / k(C) N \unlhd C N / k(C) N \in Q \mathscr{H}=\mathscr{H},
$$


we have that $k(C N) / k(C) N \in \mathscr{H}$. Consequently, $C^{*} / L \in \mathscr{H}$ by the hypothesis on $\mathscr{H}$. Since $k\left(C^{*}\right)=L, C^{*}$ is a completion of $M$ such that $N \leq C^{*}$. In the case $C$ subnormal in $G$, we argue in a similar way.

Corollary 2. Let $\mathscr{H}$ be as above. Let $I(\mathscr{H})=(G$ : for each maximal subgroup $M$ of $G$, there exists $C \in I(M)$, maximal in $I(M)$ with $C / k(C) \in \mathscr{H})$ and $S(\mathscr{H})=(G$ : for each maximal subgroup $M$ of $G$, there exists $C \in S(M)$ with $C / k(C) \in \mathscr{H})$. Then $I(\mathscr{H})$ and $S(\mathscr{H})$ are saturated homomorphs.

Proof. We prove only that $I(\mathscr{H})$ is a saturated homomorph. In the case of $S(\mathscr{H})$, the arguments are quite similar. Assume that $G \in I(\mathscr{H})$ and $N \unlhd G$. Let $M / N$ be a maximal subgroup of $G / N$. Since $M$ is a maximal subgroup of $G$ and $G \in I(\mathscr{H})$, there exists a maximal completion $C$ of $M$ in $G$ such that $C / k(C) \in \mathscr{H}$. By Proposition 1, we can assume that $N \leq C$, and then it is clear that $C / N$ is a maximal completion of $M / N$ in $G / N$, and $(C / N) / k(C / N) \in \mathscr{H}$. Therefore, $G / N \in I(\mathscr{H})$.

Finally, it is rather easy to see that $I(\mathscr{H})$ is saturated.

In the final step of an induction argument there frequently appear primitive groups. Recall that a primitive group is a group $G$ with a core-free maximal subgroup $U$. A primitive group is of one of the following types:

(1) $\operatorname{Soc}(G)$, the socle of $G$, is an abelian minimal normal subgroup of $G$ complemented by $U$.

(2) $\operatorname{Soc}(G)$ is a nonabelian minimal normal subgroup of $G$.

(3) $\operatorname{Soc}(G)$ is the direct product of the two minimal normal subgroups of $G$ which are both nonabelian and complemented by $U$.

The next results will turn out to be crucial in the proof of some of our results.

Lemma (Lafuente [8]). If $G$ is a primitive group of type 2 such that there exists a core-free subgroup $M$ of $G$ complementing $\operatorname{Soc}(G)$, then $M$ is again a primitive group of type 2 and the simple component of $\operatorname{Soc}(G)$ is isomorphic to a section of the simple component of $\operatorname{Soc}(M)$.

Theorem (Baumann [3]). Let $G$ be a nonsoluble group with a nilpotent maximal subgroup. Then $O^{2}(G / F(G))$ is the product of simple groups whose Sylow 2subgroups are dihedral. Furthermore,

(i) $O_{2^{\prime}}(G)$ is nilpotent and is centralized by a Sylow 2-subgroup of $G$.

(ii) $O^{2}(G / F(G))$ is a direct product of groups isomorphic to $\operatorname{PSL}(2, q)$ with $q=2^{n} \pm 1>5$.

(iii) $O^{2}(G / F(G))$ is a minimal normal subgroup of $G / F(G)$.

\section{THE PROOFS}

Proof of Theorem 1. Assume that for each maximal subgroup $M$ of a group $G, S(M)$ contains an element $C$ with $C / k(C)$ cyclic. We see that $G$ is supersoluble by induction on $|G|$. Let $N$ be a minimal normal subgroup of $G$. It is clear that $G / N$ verifies the above property. By induction, $G / N$ is supersoluble. Since the class of all supersoluble groups is a saturated formation, we have that $G$ is a monolithic primitive group. Let $M$ be a core-free maximal subgroup of $G$, and let $C$ be a subnormal completion of $M$ with $C / k(C)$ cyclic. Now, $k(C) \leq M_{G}=1$. Hence, $C$ is a subnormal cyclic subgroup of 
$G$, and then $1 \neq C \leq F(G)$. Therefore $N=\operatorname{Soc}(G)$ is abelian, and $C \leq N$. Since $M C=G=M N$, we have $C=N$. Therefore, $G$ is supersoluble.

Proof of Theorem 2. Denote by $E_{\mathscr{Z}}$ the Schunck class of all groups whose composition factors are in $\mathscr{X}$. Let $G$ be a minimal counterexample to the theorem. Since the class of nilpotent groups is a saturated Fitting formation, we can apply Proposition 1 and assume that $G$ is a monolithic primitive group and $N=\operatorname{Soc}(G) \notin E_{\mathscr{X}}$. Let $M$ be a core-free maximal subgroup of $G$. There exists a maximal completion $C$ of $M$ in $G$ such that $C / k(C)$ is nilpotent. Since $k(C) \leq M_{G}=1, C$ itself is nilpotent. Take $L \leq G$ such that $C$ is a maximal subgroup of $L$. By Baumann's theorem, $L \in E_{\mathscr{Z}}$. Maximality of $C$ gives $k(L) \not \leq M$, and hence $N \leq k(L)$. Therefore $N \in E_{\mathscr{Q}}$, final contradiction.

Proof of Theorem 3. Assume that $G$ is not $\pi$-soluble, and let $G$ be a minimal counterexample. Suppose that $H_{G} \neq 1$, and let $N$ be a minimal normal subgroup of $G$ such that $N \leq H$. Clearly, the hypotheses of the theorem are satisfied in $G / N$. By minimality of $G, G / N$ is $\pi$-soluble. Now, $N$ is $\pi$-soluble since $N \leq H$. Therefore $G$ is $\pi$-soluble, a contradiction. Consequently, we can assume $H_{G}=1$ and $G$ is a primitive group. Since the class of all $\pi$-soluble groups is a saturated formation, we can assume that $G$ is a primitive group of type 2. Since $N=\operatorname{Soc}(G)$ is a completion of $H$ in $G$, and since $N$ is not $\pi$-soluble, we have that $N \cap H=1$. Applying Lafuente's result, $H$ also is a primitive group of type 2, and the simple component of $\operatorname{Soc}(G)$ is an epimorphic image of the simple component of $\operatorname{Soc}(H)$. This implies $\operatorname{Soc}(G)$ is $\pi$-soluble, the final contradiction.

Proof of Theorem 4. First, we prove that $S(G)$ is soluble by induction on $|G|$. It is not difficult to see that $S(G) N / N \leq S(G / N)$ for each normal subgroup $N$ of $G$. Therefore, we can assume that $G$ is a monolithic primitive group, $A=\operatorname{Soc}(G) \leq S(G)$, and $S(G) / A$ is soluble. Now, if $G$ is a primitive group of type $1, A$ is soluble. Then, $S(G)$ is soluble and we are done. Therefore, we may suppose that $G$ is a primitive group of type 2, i.e., $\operatorname{Soc}(G)$ is a nonabelian group. Let $T$ be a $c$-maximal subgroup of $G$ with $T_{G}=1$ (see [1]). Then $G=T A$ and $T \in \mathcal{J}(G)$ by [5, Theorem 2]. This is a contradiction since $A \leq T$.

Consequently, $S(G)$ is soluble. Now, each normal soluble group of $G$ is contained in $S(G)$. Therefore, $S(G)$ is the soluble radical of $G$.

Proof of Theorem 5. (i) Suppose, first, that the Sylow $p$-subgroups of $G$ are abelian. We use induction on $|G|$. If $N$ is a minimal normal subgroup of $G$ and $M / N$ is a maximal subgroup of $G / N$ that has no maximal completion $C / N$ with $(C / N) / k(C / N)$ abelian, by Proposition $1, M$ is a maximal subgroup of $G$ and fails to have a maximal completion $D$ with $D / k(D)$ abelian. Therefore each member of $\mathscr{A}(G / N)$ is $p$-nilpotent and the Sylow $p$-subgroups of $G / N$ are abelian. By minimal election of $G$, we have that $G / N$ is $p$-soluble. Since the class of all $p$-soluble groups is a saturated formation, we may assume that $G$ is a monolithic primitive group. Denote by $N=\operatorname{Soc}(G)$ the unique minimal normal subgroup of $G$. It is clear that we can suppose $p$ divides the order of $N$. Let $P$ be a Sylow $p$-subgroup of $N$. If $N_{G}(P)=G$, then $N$ is a $p$-group and we are done. Otherwise, let $M$ be a maximal subgroup of $G$ such that 
$N_{G}(P) \leq M$. Then $G=M N$ and $M \in A(G)$ by [5, Theorem 2]. So, $N_{G}(P)$ is $p$-nilpotent. Since $P$ is abelian, $P \leq Z\left(N_{G}(P)\right)$. By a well-known theorem of Burnside, $N$ is $p$-nilpotent, a contradiction.

(ii) Suppose now that $p$ is an odd prime. Arguing by induction on $|G|$ with arguments similar to those used in (i), we can assume that $G$ is a monolithic primitive group and $p$ divides the order of $\operatorname{Soc}(G)$. Let $T=\operatorname{Soc}(G)$. Suppose that $T$ is nonabelian. Let $P$ be a Sylow $p$-subgroup of $T$. Let $J(P)$ be the Thompson subgroup of $P$ and $X=N_{G}(Z(J(P)))$. Clearly, $N_{G}(P) \leq X$. If $X=G$, then $T \leq Z(J(P))$, and $T$ is abelian, a contradiction. Thus, $X<G$, and $G=N_{G}(P) T=X T$. Let $M$ be a maximal subgroup of $G$ such that $X \leq M$. Since $M \in \mathscr{A}(G) . M$ is $p$-nilpotent. Consequently, $N_{G}(Z(J(P)))$ is $p$-nilpotent. Applying the Glauberman-Thompson normal $p$-complement theorem (see [6, p. 280]), we have that $G$ is $p$-nilpotent, a contradiction. Thus, $G$ is $p$-soluble.

\section{REFERENCES}

1. A. Ballester-Bolinches, Maximal subgroups and formations, J. Pure Appl. Algebra 61 (1989), 223-232.

2. __ On the intersection of families of maximal subgroups of a finite group, J. Pure Appl. Algebra 67 (1990), 101-109.

3. B. Bauman, Endliche nichtauflösbare Gruppen mit einer nilpotenten maximalen Untergruppe, J. Algebra 38 (1976), 119-135.

4. W. E. Deskins, On maximal subgroups, Proc. Sympos. Pure Math., vol. 1, Amer. Math. Soc., Providence, R.I., 1959, pp. 100-104.

5. __ A note on the index complex of a maximal subgroup, Arch. Math. (Basel) 54 (1990), 236-240.

6. D. Gorenstein, Finite groups, Chelsea, New York, 1967.

7. B. Huppert, Endliche Gruppen I, Springer-Verlag, Berlin and New York, 1968.

8. J. Lafuente, Eine Note über nichtabelsche Hauptfaktoren und maximale Untergruppen einer endliche Gruppe, Comm. Algebra 13 (1985), 2025-2036.

9. J. Rose, The influence on a group of its abnormal structure, J. London Math. Soc. (2) 40 (1965), 348-361.

Departament d'Algebra, Universitat de Valencia, C/ Dr. Moliner 50, 46100 BurjasSot, Valencia, Spain

Departamento de Matemática e Informática, Universidad Pública de Navarra, "El Sario," C/ El Sadar s/n, 31006 Pamplona, Spain 